chercher : repérer : avancer

Cet article est disponible en ligne à l'adresse :

http://www.cairn.info/article.php?ID_REVUE=OUTE\&ID NUMPUBLIE=OUTE_020\&ID ARTICLE=OUTE_020 0151

\title{
Inégalités régionales et rébellions au Soudan
}

\section{par Éric DENIS}

\author{
érès | Outre - Terre
}

$2007 / 3-n^{\circ} 20$

ISSN ???| ISBN 978-2-7492-0924-1| pages 151 à 168

Pour citer cet article :

- Denis n, Inégalités régionales et rébellions au Soudan, Outre - Terre 2007/3, n²0, p. 151-168.

Distribution électronique Cairn pour érès.

(C) érès. Tous droits réservés pour tous pays.

La reproduction ou représentation de cet article, notamment par photocopie, n'est autorisée que dans les limites des conditions générales d'utilisation du site ou, le cas échéant, des conditions générales de la licence souscrite par votre établissement. Toute autre reproduction ou représentation, en tout ou partie, sous quelque forme et de quelque manière que ce soit, est interdite sauf accord préalable et écrit de l'éditeur, en dehors des cas prévus par la législation en vigueur en France. Il est précisé que son stockage dans une base de données est également interdit. 


\section{Inégalités régionales et rébellions au Soudan ${ }^{1}$}

Éric Denis ${ }^{2}$

\section{Coup de force pour la justice et l'égalité}

Le 10 mai 2008, le Mouvement pour la justice et l'égalité (JEM), le plus puissant des fronts rebelles du Darfour, fort d'une logistique impeccable et d'une très solide expérience des attaques éclairs, réussit à lancer depuis le Tchad et le NordDarfour une colonne de quelque 100 technicals - ces Toyota Land Cruiser parfois assortis d'une puissante mitrailleuse russe - et pas moins d'un millier de combattants sur les faubourgs ouest de Khartoum, déjouant tous les dispositifs de sécurité. Les combats se sont prolongés toute la journée dans les rues d'Omdurman, faisant de nombreuses victimes parmi les rebelles et tuant aussi des civils et une centaine de membres des services de sécurité.

Les destructions et les morts d'Omdurman ont transposé le tragique conflit du Darfour au cœur du Soudan. La prospérité pétrolière et affairiste de Khartoum de même que l'apparente solidité du régime en sortent ébranlées. La Sécurité nationale a préféré cantonner l'armée dans ses casernes par manque de confiance à l'égard de garnisons largement recrutées parmi les gens de l'Ouest ; des soldats plus généralement en attente d'arriérés de solde considérables qui ont depuis l'automne 2007 manifesté à plusieurs reprises dans les rues de Khartoum et bloqué les ponts reliant Omdurman à la capitale. La crainte d'un retournement des troupes qui aurait pu transformer ce raid en putsch a été grande.

Comble de l'humiliation pour le régime, le leader du JEM a réussi à rejoindre sain et sauf ses bases arrière au Nord-Darfour après l'attaque. Dans ses premières déclarations, Khalīl Ibrāhīm va rappeler que le conflit du Darfour n'est pas résolu et que l'exigence quant aux « droits des populations marginalisées au Darfour et

1. J'emprunte ici le titre de l'article de D. Roden, « Regional Inequality and Rebellion in the Sudan », Geographical Review, vol. 64, n 4, p. 498-516, 1974, pour souligner l'extrême pertinence quasi-visionnaire de cette analyse d'une marginalité régionale structurelle et pérennisée au Soudan, voire son aggravation.

2. Chercheur au SEDET-CNRS-Paris 7. 
partout ailleurs au Soudan » demeure (17 mai 2008).

Notre propos est ici d'esquisser un bref tableau de la confiscation de la représentation politique du fait de quelques ethnies de la vallée du Nil et du Nord, tout comme de l'extrême injustice dans la redistribution régionale des richesses et de l'offre de services au Soudan avec de profondes inégalités régionales que ce soit en matière de santé, d'accès à l'eau ou à l'éducation. Ce processus de marginalisation est d'autant plus important qu'il constitue le fondement commun, sans cesse réactivé et mobilisé, du sentiment de relégation partagé par toutes les communautés des régions périphériques. La marginalisation politique, sociale, économique, mais aussi culturelle n'est pas le lot des seules populations du Darfour. Les rebelles de l'Ouest n'ont d'ailleurs de cesse de le rappeler dans une perspective d'adhésion plus large à leur revendications et mobilisation. De fait, fronts et oppositions coexistent et ont coexisté dans toutes les provinces du Soudan.

\section{Une représentation ethnocratique étroite}

Le JEM n'est pas le premier mouvement du Darfour à prendre les armes début 2003. La Sudan Liberation Movement/Army (SLM/A), davantage portée par une majorité de Four mettant plutôt en avant les questions identitaires et de justice de la représentation politique que les injustices sociales, a conduit les premières attaques, notamment celles contre la garnison et l'aéroport d'El-Fasher en avril.

Khalīl Ibrāhīm reste proche de la mouvance islamiste d'Hassan al-Turabi, ex-mentor du régime du président Omar al-Bashir et écarté du fait de la négociation qui aboutira début 2005 à la signature des accords de Naivasha entre le Nord et le Sud. Le régime ayant été amené par le 11 Septembre et les avancées de fin 2002 à prendre ses distances avec la mouvance islamiste internationaliste ; ceci au profit exclusif d'affairistes surtout intéressés à engranger les dividendes de leur engagement à la tête du régime au moment où les revenus pétroliers commencent à affluer.

Cette exclusion a coïncidé avec la sortie d'un ouvrage anonyme, le Livre noir, vite interdit mais distribué à la sortie des mosquées de la capitale. Il va très largement circuler. Sur une centaine de pages, il énumère de façon très clinique et objective les profondes inégalités régionales, culturelles, politiques et sociales qui affectent le Soudan. Il s'inscrit dans une approche de terrain en faveur de la justice sociale et de l'égalité régionale que prônent les islamistes. 


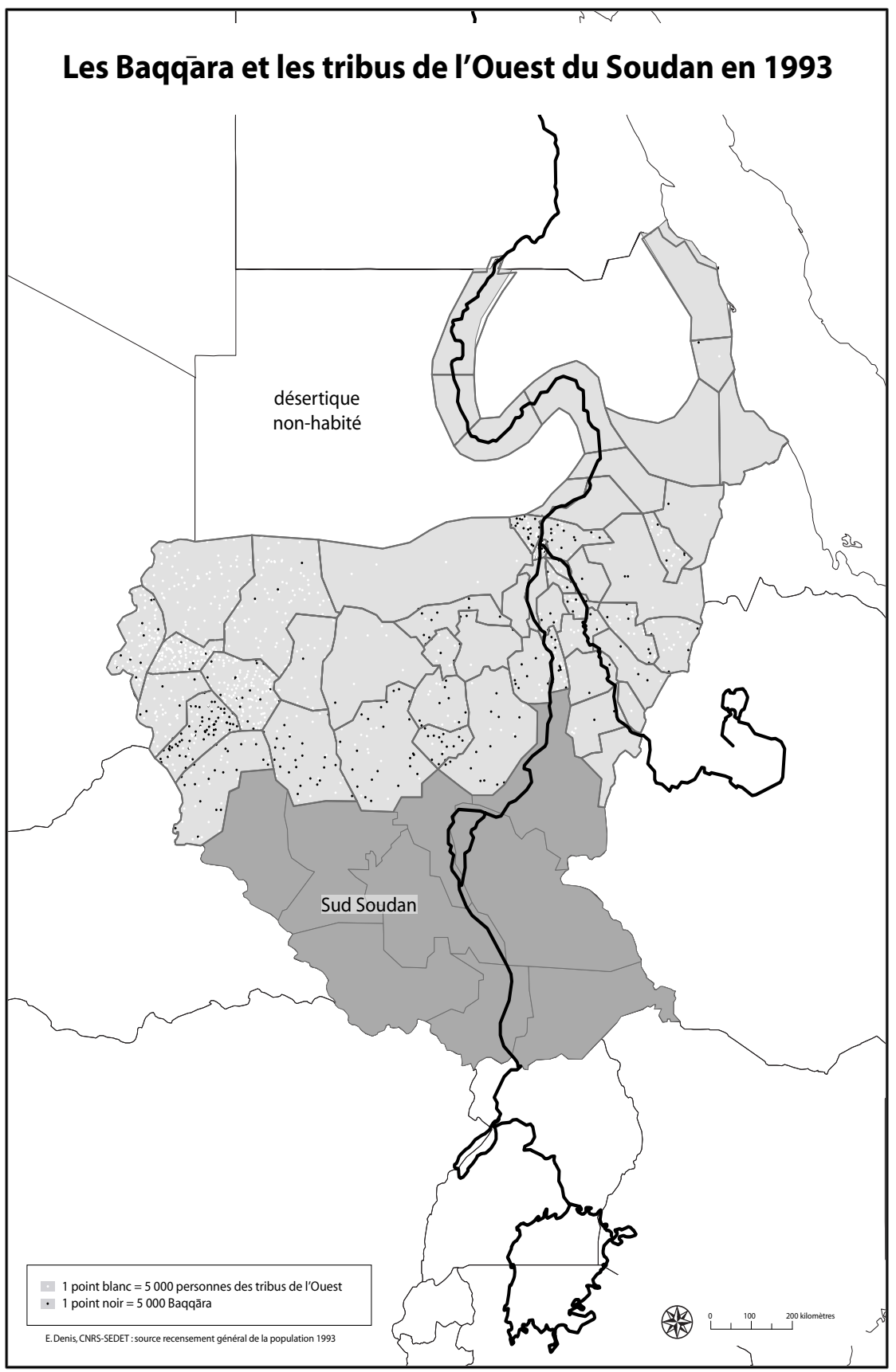


La rupture de ces derniers avec le régime tient largement au déni de leaders affairistes, opposés à la redistribution vers les régions marginalisées, et qui ont réduit de façon drastique les dépenses sociales. S'en sont suivies diverses tentatives des islamistes pour reprendre la main auprès des marginaux déçus par les régressions continues en matière de justice sociale et spatiale, alors même que le régime de 1989 avait affiché des ambitions très fortes, à ses débuts ; il voulait marquer un changement radical après la période dite démocratique qui avait été aussi et avant tout celle d'un mahdisme (du Mahdi de la reconquête et de la première unification théocratique du Soudan : 1885-1898) proarabe ; ce qui avait dans les premiers temps assuré à l'inqaz, au régime du « salut » islamiste une adhésion forte notamment des populations non-arabes de l'Ouest. Une manipulation des populations des régions marginales qui resta, comme avant 1989, sans suite en termes de redistribution et de développement périphériques.

Pour partie, le JEM est devenu le bras armé de ces populations marginalisées. Il a réactualisé le discours mobilisateur des islamistes tout en assurant une autonomie d'action et de négociation à la mouvance des pasteurs zaghāwa dans la lutte des habitants de l'Ouest contre le régime, même si le JEM est loin d'être composé uniquement de Zaghāwa. Après le Darfur Peace Agreement de mai 2006 signé à Abuja avec une partie du SLM, la résistance a implosé. Le JEM devenant alors le plus puissant des mouvements rebelles de l'Ouest.

De fait, le Livre noir dont on peut lire une traduction anglaise partielle sur le site du JEM ${ }^{3}$ dénonce d'une façon argumentée des injustices criantes et structurelles. En 2000, l'encadrement des institutions fédérales - gouverneurs, ministres des États fédéraux et district commissioners (tableau 1) - est assuré pour plus de la moitié par des cadres originaires des deux États de la vallée du Nil au nord de Khartoum, Nil (Ed-Damer) et Nord (Dongola). L'enjeu est de taille : ce verrouillage sous-tend la maîtrise de la redistribution des postes et des richesses, des terres et des équipements, mais aussi le contrôle de la collecte des impôts et des taxes locales.

\begin{tabular}{|l|c|c|c|c|c|c|}
\hline \multicolumn{7}{|c|}{ Tableau 1 : L'origine des cadres de l'administration régionale en 2000} \\
\hline Région & Gouverneurs & Commissioners & \multicolumn{2}{c|}{ Ministres } \\
\hline & No. & $\%$ & No. & $\%$ & No. & $\%$ \\
\hline Nord & 9 & 56,3 & 160 & 50,6 & 240 & 47,5 \\
\hline Est & 2 & 12,5 & 13 & 4,1 & 9 & 1,8 \\
\hline Centre & 2 & 12,5 & 27 & 8,5 & 25 & 5 \\
\hline Sud & Exclus & Exclus & 69 & 21,8 & 160 & 31,7 \\
\hline Ouest & 3 & 18,8 & 47 & 14,9 & 71 & 14,1 \\
\hline Total & 16 & 100 & 100 & 100 & 505 & 100 \\
\hline Adapté du Livre Noir, 2000 (données vérifiées par ailleurs) \\
\hline
\end{tabular}

3. $<$ http://sudanjem.com/>. 
Il ne s'agit pas d'une réalité nouvelle, mais bien d'une rente qui se reproduit au moins depuis l'indépendance du Soudan en 1956 et la constitution de son premier gouvernement.

\begin{tabular}{|c|c|c|c|c|c|c|c|c|c|c|}
\hline \multicolumn{10}{|c|}{ Tableau 2 Répartition des postes ministériels depuis 1954 en fonction de l'ori- } \\
gine régionale des ministres
\end{tabular}

Cette monopolisation, récurrente depuis l'indépendance, des positions de pouvoir ne correspond nullement à la distribution démographique régionale. Elle témoigne, sans détour, d'un rapport de domination qui s'inscrit dans une structure à partir de laquelle le contrôle ancien du pôle central historique, la vallée et Khartoum où se concentrent capital économique et culturel, permet de reproduire et renforcer une hégémonie sur l'espace soudanais sans que puisse jamais émerger une alternative périphérique.

Les populations de la vallée du Nil au nord de Khartoum ne représentent pas plus de $5 \%$ des Soudanais et même si nous leur additionnons la moitié environ des habitants de Khartoum d'origine nordiste, leur hégémonie politique et économique ne repose pas sur la masse démographique nécessaire. En revanche, le Darfour avec près de 7,5 millions d'habitants dénombrés en 2002 constitue un môle démographique majeur que seul le rattachement de la totalité des populations de l'Entre-deux-Nils - soit l'ensemble formé par Gezira, Nil-Blanc et Sennar - aux populations du Nord permettrait de contrebalancer. L'Ouest - Darfour plus Kordofan - soit quelque 11 millions d'habitants, est de loin la masse démographique la plus importante en périphérie. Et c'est bien là que réside l'un 
des dangers majeurs du conflit au Darfour : ce dernier n'est en définitive pas si marginal et la guerre, comme en témoignent les événements récents, peut être portée jusqu'à Khartoum.

\begin{tabular}{|c|c|c|c|c|c|c|c|c|c|c|c|c|c|}
\hline \multicolumn{14}{|c|}{ Tableau 3 Distribution régionale de la population 1956-2002 } \\
\hline & \multicolumn{6}{|c|}{ Nombre d'habitants en milliers } & \multicolumn{6}{|c|}{$\begin{array}{c}\text { Répartition régionale de la } \\
\text { population }(\%)\end{array}$} & \multirow{2}{*}{$\begin{array}{l}\text { Taux annuel } \\
\text { de variation } \\
1955-2002\end{array}$} \\
\hline & 1956 & 1965 & 1973 & 1983 & 1993 & 2002 & 1956 & 1965 & 1973 & 1983 & 1993 & 2002 & \\
\hline Vallée - Nord & 873 & 922 & 966 & 1,083 & 1,293 & 1,58 & 8.5 & 7.4 & 6.5 & 5.3 & 5.1 & 4.8 & 1.27 \\
\hline Khartoum & 505 & 793 & 1,16 & 1,802 & 3,512 & 4,574 & 4.9 & 6.3 & 7.8 & 8.8 & 13.7 & 13.8 & 4.80 \\
\hline $\begin{array}{l}\text { Centre - } \\
\text { Entre- deux- } \\
\text { Nils }\end{array}$ & 2,07 & 2,583 & 3,113 & 4,013 & 5,433 & 7,167 & 20.2 & 20.7 & 20.9 & 19.5 & 21.2 & 21.6 & 2.68 \\
\hline Est & 941 & 1,238 & 1,559 & 2,208 & 3,067 & 3,67 & 9.2 & 9.9 & 10.5 & 10.7 & 12.0 & 11.1 & 2.94 \\
\hline Kordofan & 1,762 & 1,996 & 2,217 & 3,093 & 3,323 & 3,772 & 17.2 & 16.0 & 14.9 & 15.0 & 13.0 & 11.4 & 1.63 \\
\hline Darfour & 1,329 & 1,735 & 2,173 & 3,093 & 4,638 & 7,405 & 13.0 & 13.9 & 14.6 & 15.0 & 18.1 & 22.3 & 3.72 \\
\hline $\begin{array}{l}\text { Total Nord- } \\
\text { Soudan }\end{array}$ & 7,48 & 9,268 & 11,188 & 15,293 & 21,267 & 28,168 & 73.1 & 74.2 & 75.2 & 74.4 & 83.1 & 84.9 & 2.86 \\
\hline Sud Soudan & 2,752 & 3,224 & 3,684 & 5,271 & 4,321 & 5 & 26.9 & 25.8 & 24.8 & 25.6 & 16.9 & 15.1 & 1.28 \\
\hline Total Soudan & 10,232 & 12,492 & 14,872 & 20,564 & 25,588 & 33,168 & 100 & 100 & 100 & 100 & 100 & 100 & 2.53 \\
\hline
\end{tabular}

D'autant que le Nord lui-même n'est pas unifié et qu'il a lui aussi ses logiques internes de marginalisation et de fronts. Les Nubiens, Maha en particulier, de l'extrême Nord du pays - entre Wadi-Alfa à la frontière égyptienne et Dongola affichent de plus en plus ouvertement leur opposition radicale au régime et affirment leur différence culturelle, d'aucuns envisageant même le recours à la lutte armée.

Dans la vallée, à 350 kilomètres au nord de Khartoum, les populations directement affectées par la mise en eau du barrage hydroélectrique de HamdabMérowé ${ }^{4}, 50000$ environ, se sont fermement opposées aux méthodes de déplacement et de compensation, ce qui a provoqué des heurts d'une extrême violence. La fronde était motivée par les injustices criantes en matière de compensation, de redistribution des terres et d'indemnités entre les tribus arabes. Elles ont été très défavorables aux Manāsīr, Amri et Hamadad et interprétées comme une

4. D’une capacité de $1250 \mathrm{MW}$, le barrage de Mérowé doit dès l'an prochain augmenter de $150 \%$ la production électrique du Soudan et des périmètres irrigués y sont associés. Les études du barrage de Mérowé implanté à proximité de la 4e cataracte ont été conduites par un bureau d'étude allemand, sa construction est chinoise et les turbines ont été produites par Alstom, $<$ www.merowedam.gov.sd/>. 
appropriation foncière au profit des Ja'aliyin, une des deux tribus dominantes de l'amont du barrage avec les Shayqiya à laquelle appartient le président et général Omar al-Bashir et dont sont également issues les grandes familles aux affaires depuis l'indépendance. Les populations affectées par le barrage de Kabjar en chantier plus au nord ont aussi manifesté, mais se sont vues confrontées à une répression capable d'aller aux extrémités pour que l'onde des protestations ne gagne pas du terrain (4 manifestants tués en juin 2007) ${ }^{5}$.

Diviser, opposer, c'est évidemment la stratégie adoptée au Darfour depuis des décennies par le maintien, voire le renforcement constant, des clivages entre les éleveurs baqqāra qui se revendiquent d'une ascendance arabe plus anciennement enracinée dans la région, sur les versants du Jebel Marra qui culmine à 3000 mètres. L'entrée en résistance de cette dernière en 2003 a déclenché une vague de répression, de razzias, de meurtres et de déplacements massifs de population qui a encore accentué la distance culturelle et creusé l'antagonisme entre les géographies ${ }^{6}$. Les milices supplétives arabes sont devenues le bras armé d'une conquête territoriale alors que la démographie des populations arabes, moins vigoureuse que celle des Four et autres tribus « natives », ne permettait plus de contrôler le Darfour ${ }^{7}$. À l'été 2006, les Arabes Abbala du Nord-Darfour aimaient cependant à dire que la guerre était finie car les chameaux avaient mangé les mangues du Jebel Marra.

Au total, les populations des tribus de l'Ouest non-arabes peuvent être estimées en 2005 à quelque 5 millions, contre 922000 Arabes Baqqāra et 300000 autres Arabes originaires de la vallée du Nil - notamment des commerçants et des fonctionnaires ${ }^{8}$. Dans ces conditions, pour conserver l'hégémonie dans les urnes, il est essentiel d'une part de jouer la carte de la concentration sur des territoires précis, et, d'autre part, de pousser par la force les tribus du Darfour à quitter certaines localités ou pire de les faire disparaître. C'est pourquoi le conflit prend de plus en plus la forme d'une guerre de déracinement où les milices janjawid

5. Vidéo de la manifestation du 13 juin 2008 (45 mn) sur <http://video.google.com/ videoplay?docd $=-3249955364069010660 \& \mathrm{hl}=\mathrm{en}>$.

6. Cf. J. Flint, A. de Waal, Darfur : A Short History of a Long War, Londres, Zed Books, 2005.

7. R. A. Henin, « The Patterns and Causes of Fertility Differentials in the Sudan », Population Studies, vol. 23, n² 2, p. 171-198, 1969, exposait déjà un différentiel de fécondité défavorable aux populations non-sédentaires ; il s'était en particulier intéressé aux Baqqāra du Darfour.

8. L'enquête sur les communautés de pasteurs du Darfour conduite par l'association al-Massar Charity Organization for Nomads Development \& Environmental Conservation entre janvier et mars 2003 donne une population d'environ 850000 pasteurs arabes baqqāra (cf. tableau 3) qui corrobore notre estimation basée sur une projection des données censitaires et en confirme la cohérence à partir d'une démarche statistique complètement différente, voire d'un programme pro-Baqqāra. 
ne cherchent pas forcément à faire beaucoup de morts lors de leurs razzias. Elles tuent les chefs et les anciens, ceux qui sont dépositaires de la mémoire collective, des droits d'usage du sol et des règles de résolution des conflits. Elles chassent les populations et empêchent leur retour en empoisonnant parfois les sources. Elles tendent systématiquement à déraciner les communautés non-arabes, à les forcer à abandonner leur terre pour le bush où ces dernières vont errer, vivre de cueillette et parfois mourir affaiblies par le manque de nourriture et les blessures alors que la possibilité du retour dans les villages est interdite par des patrouilles de miliciens et quelquefois l'établissement de familles d'agro-pasteurs baqqāra, voire de populations arabes de l'Est du Tchad. La plupart finissent par rejoindre les camps et s'urbanisent peu à peu, perdant tout espoir d'un retour. Là encore, à l'échelle du Darfour, la relation incohérente entre le poids démographique et la domination politique locale combinée à une pression accrue sur la maîtrise des ressources, du sol et de l'eau ne pouvait se liquider que dans le sang, le pouvoir étatique détenteur de légitimité se maintenant lui-même en place par les armes.

\begin{tabular}{|c|c|c|c|c|c|c|c|}
\hline \multicolumn{8}{|c|}{$\begin{array}{l}\text { Tableau } 4 \text { Distribution des populations des tribus Baqqāra* et des tribus de } \\
\text { l'Ouest en } 1956 \text { et en } 1993\end{array}$} \\
\hline \multirow{3}{*}{$\begin{array}{l}\text { Région } \\
\text { Nord-Darfour } \\
\end{array}$} & \multirow{3}{*}{$\begin{array}{l}\text { Districts** } \\
\text { Kutum } \\
\end{array}$} & \multirow{3}{*}{\begin{tabular}{c|} 
Baqqāra \\
1956 \\
12026 \\
\end{tabular}} & \multirow{3}{*}{$\begin{array}{c}\begin{array}{c}\text { Tribus de } \\
\text { l'Ouest }\end{array} \\
1956 \\
143,023\end{array}$} & \multirow{3}{*}{\begin{tabular}{c|} 
Baqqāra \\
1993 \\
1,319
\end{tabular}} & \multirow{3}{*}{$\begin{array}{c}\begin{array}{c}\text { Tribus de } \\
\text { l'Ouest }\end{array} \\
1993 \\
383,866 \\
\end{array}$} & \multirow{2}{*}{\multicolumn{2}{|c|}{$\begin{array}{c}\begin{array}{c}\text { Taux annuel } \\
\text { de variation }\end{array} \\
1956-1993\end{array}$}} \\
\hline & & & & & & & \\
\hline & & & & & & \multicolumn{2}{|c|}{\begin{tabular}{l|l}
-5.80 & 2.70 \\
\end{tabular}} \\
\hline & El-Facher & 3,026 & 121,961 & 13,017 & 381,763 & 4.02 & 3.13 \\
\hline & Um-Kadada & 942 & 28,904 & 5,021 & 90,909 & 4.63 & 3.15 \\
\hline \multirow[t]{3}{*}{ Ouest -Darfour } & al-Geneina & 34,537 & 83,352 & 51,775 & 366,362 & 1.10 & 4.08 \\
\hline & Zalingeï & 1,518 & 94,57 & 21,597 & 505,582 & 7.44 & 4.63 \\
\hline & Wadi Salih & 6,841 & 92,062 & 23,138 & 203,821 & 3.35 & 2.17 \\
\hline \multirow[t]{5}{*}{ Sud-Darfour } & Nyala & 18,413 & 63,287 & 88,016 & 602,621 & 4.32 & 6.28 \\
\hline & al-Dayin & 79,673 & 8,834 & 115,635 & 75,145 & 1.01 & 5.96 \\
\hline & $\begin{array}{l}\text { Buram / Idd } \\
\text { al-Fursan }\end{array}$ & 111,657 & 21,303 & 403,876 & 302,064 & 3.54 & 7.43 \\
\hline & Darfour total & 270,589 & 659,252 & 725,387 & $2,914,126$ & 2.70 & 4.10 \\
\hline & $\begin{array}{l}\text { Poids des } \\
\text { populations } \\
\text { Nord- Soudan }\end{array}$ & $20.4 \%$ & $49.6 \%$ & $15.6 \%$ & $62.8 \%$ & & \\
\hline \multicolumn{8}{|c|}{$\begin{array}{l}\text { E. Denis ; source : recensements de la population de } 1956 \text { et } 1993 \\
\text { * Baqqāra et Gamala ou Abbala (pasteurs, éleveurs de vaches ou de chameaux) } \\
\text { ** limites constantes de } 1956 \text { par regroupement des unités créées depuis. }\end{array}$} \\
\hline
\end{tabular}


Le poids démographique était également au cœur des accords de paix entre le Nord et le Sud. C'est sur la base d'une population sudiste estimée à 11 millions que la répartition des postes au gouvernement et le partage des revenus (taxes et pétrole) ont été négociés. L'accord confère au Sud un tiers des postes dans l'administration, au gouvernement fédéral et à l'assemblée, ainsi que la moitié des revenus pétroliers.

Or, la rébellion qui a éclaté au Darfour se superpose aux mois de cessez-le-feu Nord-Sud et de négociations menant aux accords de Naivasha en janvier 2005. Elle se comprend donc comme une réaction visant à faire entendre la voix des marginalisés du Nord-Soudan devant un partage des richesses entre les pouvoirs issus de la guerre Nord-Sud - une répartition qui légitime et entérine le statu quo au Nord, même si les accords prévoient des élections régionales en 2009 (la constitution de partis restant verrouillée).

La « question statistique » s'est encore compliquée avec le recensement conduit en définitive fin avril $2008^{9}$. Car ses résultats seront au fondement de la répartition du pouvoir et des richesses entre Nord et Sud, du référendum d'autodétermination de 2011 au Sud et du poids des 25 régions - Nord et Sud aux élections à l'assemblée fédérale prévues pour 2009. Or, la mise en place du recensement au Sud est déjà venue confirmer qu'il n'y aurait guère plus de 5 à 6 millions de Sudistes alors que les déplacés au Nord sont venus renforcer le poids de celui-ci et que les réfugiés dans les pays frontaliers du Sud n'ont pas rejoint massivement leur communauté d'origine. L'équilibre né des accords - en particulier le partage des revenus pétroliers - pourrait qui plus est être contesté par le régime nord-soudanais. La polémique statistique n'a donc cessé de s'amplifier et devrait être d'une intensité rare quand les chiffres seront officiellement communiqués en principe au mois de novembre 2008.

Sans compter que le Darfour pourrait disposer d'une représentation législative plus importante que le Sud et que toute autre région du Soudan. D'autant que les leaders rebelles ont appelé à boycotter le recensement et qu'ils sont particulièrement suivis par les populations des camps qui n'ont pu être recensées - pas moins de 2 millions de Soudanais manquant à l'appel ${ }^{10}$. On comprend mieux au demeurant dans pareil contexte pourquoi les anciens ennemis, soit le National Congress Party (du président) et le Sudan People's Liberation Movement (SPLM) sudiste s'accordent sur le «traitement » à réserver au Darfour. Une réouverture des négociations conduirait immanquablement à un démantèlement de l'accord Nord-Sud déjà bien fragilisé. Et puis la majorité des leaders du SPLM n'apprécient pas du tout l'ouverture très tardive d'un front au Darfour. Où

9. Cf. Eric Denis, «Soudan : se compter en guerre et paix », revue en ligne Echogéo, rubrique Sur le vif 2008, mise en ligne juillet 2008, <http://echogeo.revues.org. $>$.

10. Ibid. 
étaient les Darfouriens durant toutes ces années de lutte ? Nombre des actuels rebelles combattaient même dans les milices, voire dans l'armée nord-soudanaise régulière, certes pas à l'échelle des miliciens janjawid, mais tout de même.

À l'inverse, les élites de l'Ouest pensent fin 2002, à la lumière du processus de paix qui s'amorce entre les ex-rebelles du Sud et le régime, qu'il n'est de paix et de redistribution possible au Soudan qu'avec des populations marginales engagées dans la lutte armée. L'action du JEM s'inscrit au demeurant dans une longue « tradition » de rébellions régionales visant à remettre en cause par les armes le verrouillage des postes de pouvoir tout comme la répartition de l'investissement et des richesses. Dès 1938, les non-Arabes du mouvement nordiste al-kutla alSuda entendaient faire entendre leur voix, donc avant même le Sud. Les autorités coloniales l'interdirent rapidement. En 1954, l'autorisation de partis politiques au Sud se traduisit sans délais par l'émergence d'un front d'opposition très véhément et des troubles locaux : on réclamait davantage d'attention et de moyens. Ce fut partout l'aspiration à une plus grande justice et à un fédéralisme tenant compte de la diversité qui grondait très tôt, mais elle ne fut pas entendue par les premiers gouvernements issus de l'indépendance. Au Sud, l'opposition se transforma en guerre de 1963/64 à 1973, puis à nouveau de 1986 à $2005^{11}$. À l'Est, le Congrès Beja conduisit plusieurs attaques de guérilla à partir du coup d'État de 1989 pour finalement signer un accord de paix avec le régime en $2006{ }^{12}$.

\section{Domination politique et culturelle et perpétuation des inégalités sociales}

Le Soudan, 2,5 millions de $\mathrm{km}^{2}$, territoire gigantesque, un dixième de l'Afrique, s'est consolidé autour de quelques tribus arabes dont le pouvoir et la maîtrise des richesses furent définitivement assurés durant la période angloégyptienne (1899-1956). À partir des années 1920 en particulier, se conformant au principe de l'Indirect Rule qui s'imposait dans l'ensemble de l'Empire britannique, les administrateurs du condominium mirent en place une administration " indigène ", la Native Administration. Ils instaurèrent au même moment des closed districts (zones fermées) donnant pouvoir aux gouverneurs d'interdire les échanges entre certaines régions, en particulier entre le Nord et le Sud. Cette politique des closed districts fut en partie appliquée au Darfour ${ }^{13}$.

11. Cf. D. J. Johnson, The Root Causes of Sudan's Civil Wars, James Currey, Indiana University Press, 2003.

12. Cf. C. Miller (éd.), Land, ethnicity and political legitimacy in Eastern Sudan, Le Caire/ Khartoum, CEDEJ, 2005.

13. Cf. M. W. Daly, Darfur's Sorrow. A History of Destruction and Genocide, New York, 
L'administration coloniale qui avait besoin de se « soudaniser » fit bénéficier d'abord les tribus arabes de la vallée de l'accès à l'éducation. Leurs fils étaient ainsi pendant longtemps seuls en mesure d'occuper des fonctions administratives et politiques, certes subalternes. L'objectif des gouverneurs du Soudan était de créer un corps de fonctionnaires locaux en mesure de relayer l'exploitation et le contrôle du territoire soudanais. Ils trouvèrent dans les tribus arabes (médiation égyptienne) une plus grande proximité et des facilités de collaboration comme d'adaptation, de même que ces dernières avaient constitué les relais locaux de la colonisation turco-égyptienne.

Les tribus des berges de la vallée du Nil étaient aussi les seules à disposer dès le XVIe siècle, en ce qui concerne les terres irriguées, d'une propriété privée du sol que le condominium anglo-égyptien étendit à leur profit aux villes de Dongola et Khartoum en $1899^{14}$. Cette maîtrise foncière initiale a assuré aux riverains du Nil, les awlād al-balad, une position dominante. Les régions périphériques, extérieures à la vallée du Nil, ont en revanche continué à relever de la propriété collective gérée localement. Ce qui ne permettait pas d'amorcer une dynamique endogène d'investissement. Le capital restait le bétail alors que le fructueux commerce de longue distance avait été transféré à la puissance occupante une fois placées sous tutelle les royautés sacrées du Sud et les royaumes indépendants de l'Ouest, Four et Masālīt. Ensuite, l'Unregistred Land Act de 1970, tout en réduisant les compétences du droit coutumier, transfère les terres non mises en valeur à la puissance publique et confère au pouvoir central un droit de redistribution du sol favorable aux grands investisseurs. Il accentue par là encore la spoliation des sociétés locales périphériques dont les chefs coutumiers locaux ne peuvent plus attribuer que des droits d'usage, de culture qui peuvent être au demeurant remis en cause par l'administration publique fédérale.

L'éducation est la clé majeure du processus de marginalisation dans le Soudan contemporain. En 1944, 483 des 514 élèves des rares écoles secondaires étaient originaires de la vallée au nord de Khartoum ${ }^{15}$. Le recensement de 1956 révélant qu'ils comptaient encore à cette date pour 88 \% des diplômés du secondaire. Et avec la « soudanisation » de l'administration à l'indépendance, les éduqués du Nord prirent le contrôle du pays.

La situation n'a guère évolué jusqu'à présent malgré l'ouverture d'écoles, de collèges et même d'universités de province dans tous les États du Nord depuis l'établissement du fédéralisme en 1994. Les moyens n'ont guère suivi et le fossé creusé au début du siècle est toujours aussi profond. La question est d'avoir

Cambridge University Press, 2007.

14. Cf. S. M. Ahmed el Mahdi, Introduction to the Land Law of the Sudan, Khartoum University Press, 1979.

15. M. Omar Bashir, Educational Development in the Sudan, 1898-1956, Oxford, Clarendon Press, 1969. 
des infrastructures scolaires en nombre suffisant pour couvrir des populations dispersées, et aussi les enseignants qui acceptent de s'isoler dans des petites communautés, lesquels doivent être au demeurant payés. Fin 2002 nombre d'écoles des quelque 9000 villages du Darfour n'avaient pas d'enseignants et la plupart d'entre ceux qui exerçaient devaient vivre avec des arriérés de l'ordre de six mois de salaire - lot commun de la fonction publique soudanaise. Les déplacements de population et leur regroupement dans des camps ont paradoxalement permis de recruter plus d'enseignants, grâce notamment à l'UNICEF. Il en est de même en matière de santé.

Le graphique 1 traduit la domination de la vallée et de Khartoum ; de même à l'inverse pour le total dénuement du Sud suivi par les régions de l'Ouest.

\section{Graphique 1 Enfants en fin de cycle primaire scolarisés en 2006}

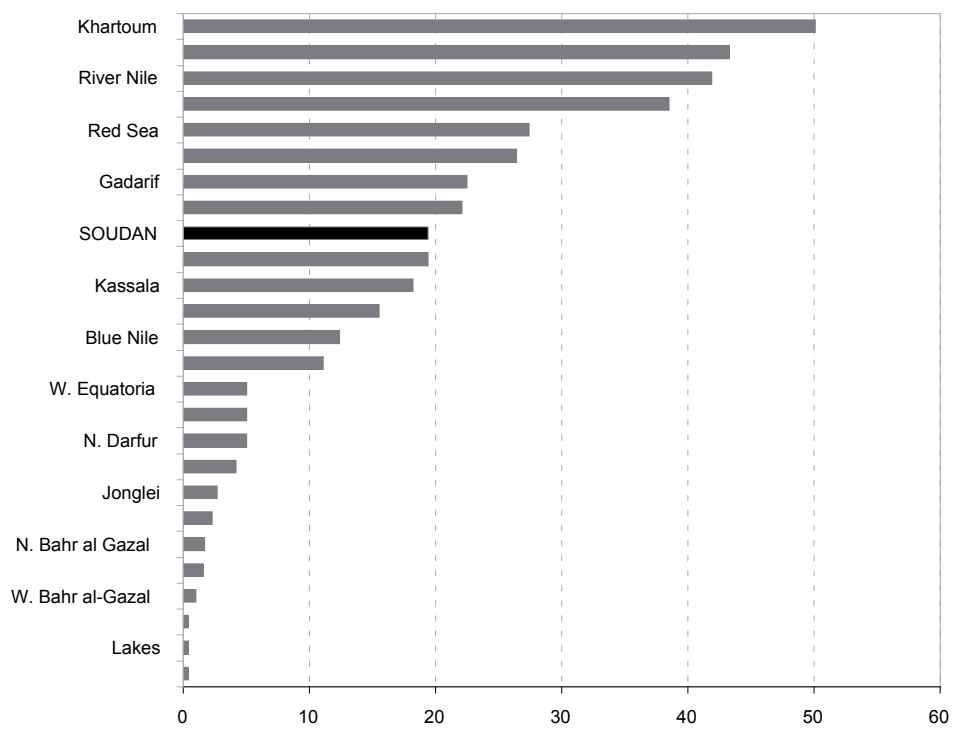

Source : SHHS, avant-projet, $2007^{16}$

16. L'enquête qui fait date Sudan Household Health Survey (SHHS), conduite tant au Sudqu'au Nord-Soudan, est la première qui couvre avec protocole uniforme la totalité du territoire. Il est donc pour la première fois possible de mesurer les inégalités sur une base unifiée. Soutenue par le United Nations Population Fund dans une perspective de mise à jour des indicateurs du Millenium Development Goal, cette enquête a été menée en partenariat par les instituts statistiques du Nord- et du Sud-Soudan. Un tirage aléatoire à partir de listes régionales de villages a produit un échantillon représentatif à l'échelle régionale de plus de 24000 familles ayant accepté de répondre au questionnaire. Notre propre suivi du terrain de la préparation et du passage en particulier au Sud nous autorise à attester de la qualité de l'information recueillie. 
Le Sud est encore presque totalement privé d'un quelconque appareil éducatif, ceci bientôt trois ans après Naivasha. La tâche de reconstruction après 25 ans de guerre apparaissant dès lors comme titanesque. On comprend mieux aussi les difficultés presque insurmontables du gouvernement du Sud-Soudan pour bâtir à partir de rien une administration publique. Avant 1956, il y avait en tout et pour tout deux écoles secondaires pour tout le Sud ; à part les missions chrétiennes qui offraient une complète éducation de base - davantage aux populations équatoriennes sédentaires qu'aux tribus de pasteurs. Dans ces conditions, la diaspora demeure la clé de la reconstruction, mais encore faut-il pouvoir l'accueillir.

C'est par le maintien de ces inégalités régionales que les héritiers du système ethnocratique ont pu conserver le pouvoir. Ils ont joué la fragmentation pour contrôler et redistribuer les richesses, soutenu les uns contre les autres, attisé les conflits locaux plutôt que cherché à les résoudre. Les milices supplétives dans les guerres tant au Sud qu'à l'Ouest relèvent de cette approche - Nuer contre Dinka au Jongleï, Arabes baqqāra contre Four et Zaghāwa au Darfour ou Murlé contre Dinka Bor... Les dépenses publiques en faveur du ministère de la Défense et de la Sécurité intérieure restant au même niveau.

Nous pourrions multiplier les exemples d'inégalités d'accès aux services de base entre régions ${ }^{17}$. Le graphique 2 ne fait que souligner l'injustice flagrante en matière d'accès à l'eau. Comme pour l'éducation, nous retrouvons les deux régions du Nord et Khartoum, puis la Gezira comme régions dont les habitants sont majoritairement à proximité immédiate de l'eau - le plus souvent par robinet dans leur cour ; au minimum par forage ou réseau local de distribution à moins de 15 minutes aller-retour. Au Sud comme à l'Ouest, mais aussi à l'Est, Kassala et Gedaref, pas moins de la moitié des familles mettent plus d'une demi-heure pour accéder à un point d'eau.

Les États où plus d'un quart des familles doivent consacrer au-delà d'une heure par jour pour aller jusqu'à un point d'eau, collecter l'eau et la rapporter incluent le Kassala (38 \%), le Jongleï (27,3\%), Lacs (35,3\%), Équatoria-Occidental (40,2 \%) et Équatoria-Central (40,6 \%). Dans Mer-Rouge, les familles consacrent en moyenne 85 minutes par jour à cette tâche, c'est dire l'absence d'équipements, de forages à l'Est que l'on retrouve au Kassala (67 minutes) et même au Nil-Blanc (65 minutes), de même que dans l'Ouest-Darfour et le Nord-Darfour où la moyenne s'établit à 45 minutes et au Sud à 58 minutes pour Équatoria-Central. On pourrait établir dans le même sens une corrélation entre ces disparités dans l'accès à l'eau et la mortalité infantile ; en ce qui concerne

17. Cf. E. Denis, « Démographie ethnique, modernisation et violence au Darfour », Annales d'Éthiopie, vol. XXI, 2005, p. 97-137. 
bien sûr les maladies hydriques, mais aussi l'accès à la médecine de base, aux traitements contre la malaria par exemple.

\section{Graphique 2 Distance à un point d'eau selon les régions en 2006}

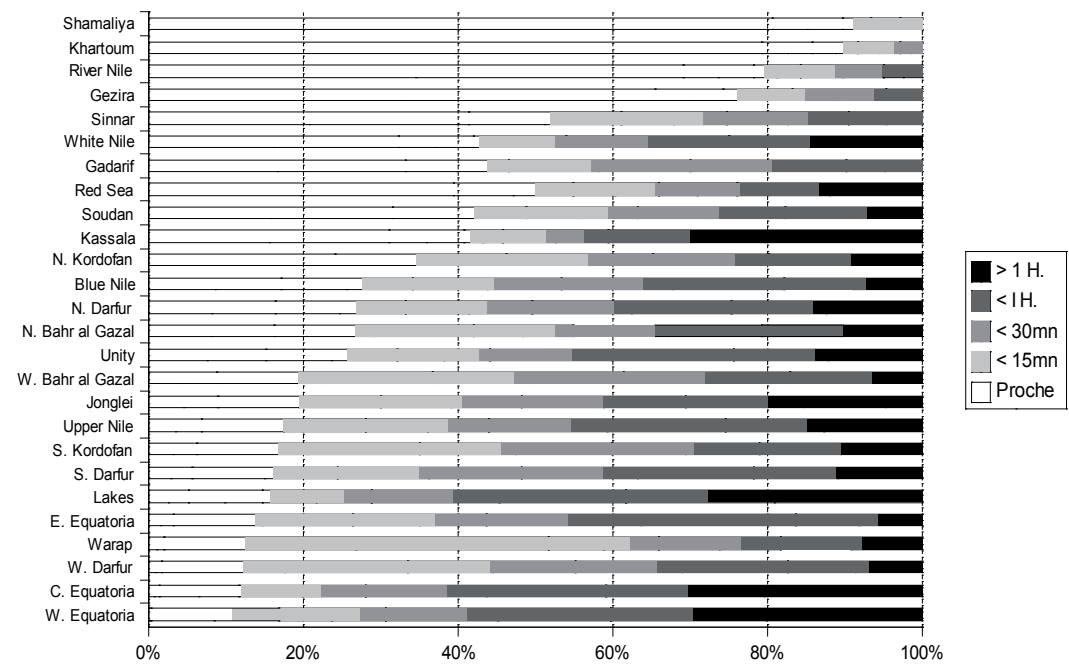

Source : SHHS avant-projet, 2007

\section{Pôles d'investissement et non-développement régional}

L'hégémonie politique à peu près sans concessions des élites des tribus arabes de la vallée du Nil s'est doublée d'une absence d'investissements dans les régions périphériques. Il n'y a eu là-bas aucune amélioration des infrastructures, voire même de profondes régressions comme au Sud avec les destructions dues à la guerre.

Depuis la colonisation et le développement du périmètre irrigué de la Gezira pour fournir en coton l'industrie textile des Midlands, la planification de l'investissement au Soudan a toujours promu une agriculture industrielle. Au début des années 1970, le pays devenait le breadbasket du monde arabe ${ }^{18}$. Outre

18. Cf. J. Kaikati, « The economy of Sudan : A Potential Breadbasket of the Arab World ? ", Int. Journal of Middle East Studies, $\mathrm{n}^{\circ} 11,1980$, p. 99-123 ; P. Oesterdiekhoff, K. Wohlmuth, "The 'Breadbasket' is empty: The options of Sudanese development policy », CJAS, $\mathrm{n}^{\circ}$ 17/1, 1983, p. 35-67. 
les nouveaux périmètres irrigués comme celui de Managīl, cela a donné plus tard ce qui reste l'un des plus grands complexes sucriers du monde, Kenana Sugar Co. Avec ses 43000 hectares de canne à sucre et sa capacité de production de 365000 tonnes de sucre par an. Un rêve de grandeur dont la faillite financière et politique est avérée, mais qui redevient une perspective d'avenir dès lors que les prix des céréales s'envolent. L'Égypte a annoncé son intention d'investir au Soudan afin d'accroître sa production céréalière, de même que l'Arabie saoudite et Abu Dhabi qui préconise avec The Arab Authority for Agricultural Investment and Development, un fonds panarabe basé à Khartoum et présent dans toute la région (notamment au Maghreb), la bonification de quelque 30000 hectares. Bien entendu, ces ambitions nouvelles ne seront pas sans menacer les pasteurs et la petite paysannerie qui ne disposent que d'un droit de passage ou d'usage du sol facile à remettre en cause.

Le boom pétrolier accompagne une industrialisation intense appuyée sur une attractivité très forte pour les investissements directs étrangers - l'une des plus fortes d'Afrique, mais elle rayonne encore moins que l'investissement agricole puisqu'elle ne concerne que Khartoum. Elle ne corrige absolument pas les inégalités du système productif ${ }^{19}$. Le Soudan, fort de joint-venture avec la Corée du Sud (Hyundai), la Chine, mais aussi l'Iran et la France (Renault Véhicules Industriels), a en effet développé de nombreuses chaînes d'assemblage dans le secteur automobile en particulier, mais aussi dans les secteurs de l'électroménager et de l'armement. Il exporte des tracteurs et des camions notamment vers l'Éthiopie voisine.

L'approche minière de l'exploitation du pays s'est doublée d'une orthodoxie financière de plus en plus marquée. Le régime de l'inqaz a fait du remboursement de la dette un nouvel instrument légitime de l'accaparement affairiste combiné à partir de 2000 avec les premières exportations de pétrole. Ainsi, le Soudan a été consacré comme l'un des meilleurs élèves du FMI en Afrique, le pays assurant par des réductions drastiques des dépenses publiques (sauf dans le secteur militaire et celui de la sécurité intérieure) un remboursement de la dette et le retour à l'équilibre des indicateurs macroéconomiques s'accompagnant d'un recul très net de l'inflation.

À partir de 1994 - mise en œuvre du fédéralisme - les régions périphériques restées extérieures aux projets stratégiques liées à l'exploitation pétrolière ou minière et à l'agriculture industrielle ont subi une nouvelle baisse de leur budget rarement compensée par des transferts du budget central (tableau 5).

L'offre de services, notamment en matière de santé, s'est encore contractée avec la réforme fédérale de 1994 pour tomber à peu près à zéro dans des régions

19. Cf. G. el-Din El Tayeb, Industry and Peripheral Capitalism in the Sudan: A geographical Analysis, Khartoum University Press, 1989. 
comme le Darfour (tableau 6). Le fédéralisme est d'autant moins une solution au développement qu'il ne s'est accompagné d'aucune démocratisation ; ce qui vaut a fortiori pour le nouvel échelon d'encadrement local des Idarah Sha abiya, jeunes cadres sans ancrage mais acquis au régime, créé pour contourner/marginaliser l'administration traditionnelle. De plus, les questions de sécurité intérieure et de stabilité demeurent la préoccupation majeure des administrations régionales. Aucune réflexion locale en matière d'investissement et de redistribution n'est possible, d'autant que les collectes potentielles d'impôt sont souvent minimales et que presque partout prévalent des situations d'urgence - ici une épidémie de méningite, là une crue destructrice, ailleurs une fièvre qui décime les troupeaux.

\begin{tabular}{|c|c|c|c|c|c|c|}
\hline & 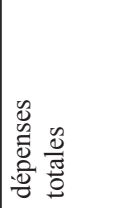 & 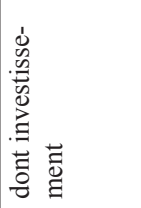 & 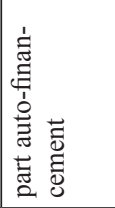 & 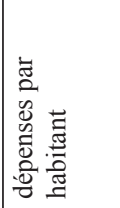 & 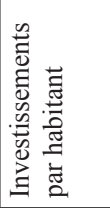 & 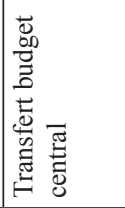 \\
\hline & millions $€$ & millions $€$ & $\%$ & $€$ & $€$ & $€ /$ hab. \\
\hline Shamaliyya & 9.65 & 0.40 & 58.9 & 15.49 & 0.65 & 6.37 \\
\hline Nahr al-Nil & 18.22 & 5.44 & 94.9 & 19.04 & 5.69 & 0.97 \\
\hline Mer Rouge & 15.44 & 1.20 & 103.8 & 17.66 & 1.38 & 0.00 \\
\hline Kassala & 13.53 & 0.05 & 68.2 & 8.88 & 0.03 & 2.82 \\
\hline Gedaref & 16.78 & 2.59 & 86.6 & 13.19 & 2.04 & 1.77 \\
\hline Khartoum & 98.06 & 13.80 & 116.4 & 21.44 & 3.02 & 0.00 \\
\hline Gezira & 40.30 & 0.57 & 33.5 & 11.08 & 0.16 & 7.37 \\
\hline Sennar & 12.28 & 0.94 & 102.3 & 9.01 & 0.69 & 0.00 \\
\hline Nil-Blanc & 6.47 & 1.06 & 158.5 & 3.97 & 0.65 & 0.00 \\
\hline Nil-Bleu & 6.02 & 0.13 & 63.8 & 11.18 & 0.24 & 4.05 \\
\hline Nord-Kordofan & 20.78 & 0.39 & 41.2 & 17.33 & 0.32 & 10.19 \\
\hline Ouest -Kordofan & 9.12 & 0.69 & 73.9 & 6.47 & 0.49 & 1.69 \\
\hline Sud -Kordofan & 3.17 & 0.00 & 43.5 & 2.72 & - & 1.54 \\
\hline Nord-Darfour & 12.08 & 0.05 & 51.4 & 5.92 & 0.03 & 2.88 \\
\hline Ouest -Darfour & 4.60 & 0.00 & 49.6 & 2.34 & - & 1.18 \\
\hline Sud-Darfour & 12.07 & 1.19 & 96.8 & 3.55 & 0.35 & 0.11 \\
\hline Nord-Soudan & 298.58 & 28.51 & 75.9 & 10.60 & 1.01 & 2.56 \\
\hline
\end{tabular}

\begin{tabular}{|l|c|c|c|c|c|}
\hline \multicolumn{2}{|c|}{ Tableau 6 Distribution des médecins en 2003 et des lits d'hôpitaux en 2000} \\
\hline & $\begin{array}{c}\text { nombre de } \\
\text { médecins }\end{array}$ & privé & public & $\begin{array}{c}\text { habitants / } \\
\text { médecin }\end{array}$ & $\begin{array}{c}\text { habitants / lit } \\
\text { hôpital }\end{array}$ \\
\hline Shamaliyya & 131 & 2,8 & 0,5 & 4756 & 434 \\
\hline
\end{tabular}




\begin{tabular}{|l|c|c|c|c|c|}
\hline Nahr al-Nil & 188 & 4 & 0,7 & 5089 & 681 \\
\hline Mer-Rouge & 103 & 2,1 & 0,6 & 8486 & 1168 \\
\hline Kassala & 195 & 3,8 & 1,5 & 7817 & 1348 \\
\hline Gedaref & 98 & 2,1 & 0,2 & 12979 & 1346 \\
\hline Khartoum & 3893 & 54 & 87,8 & 1175 & 866 \\
\hline Gezira & 571 & 11,7 & 3,2 & 6368 & 1365 \\
\hline Sennar & 220 & 4,5 & 1,1 & 6195 & 1287 \\
\hline Nil-Blanc & 171 & 3,3 & 1,3 & 9527 & 1339 \\
\hline Nil-Bleu & 65 & 1,4 & 0,1 & 8286 & 2033 \\
\hline Nord-Kordofan & 181 & 3,5 & 1,6 & 6624 & 923 \\
\hline Ouest-Kordofan & 41 & 0,9 & 0 & 34364 & 2340 \\
\hline Sud-Kordofan & 38 & 0,7 & 0,3 & 30647 & 2275 \\
\hline Nord-Darfour & 84 & 1,9 & 0 & 24303 & 3685 \\
\hline Ouest-Darfour & 26 & 0,6 & 0,1 & 75574 & 8972 \\
\hline Sud-Darfour & 133 & 2,6 & 1 & 25554 & 5636 \\
\hline Nord-Soudan & 6138 & 100 & 100 & 4589 & 1413 \\
\hline $\begin{array}{l}\text { Source : Inventaire communal 2003 \& Statistical Yearbook 2000; Bureau central des statistiques, } \\
\text { Khartoum }\end{array}$ &
\end{tabular}

Pas ou pratiquement pas de médecins, des hôpitaux saturés et peu nombreux, des dispensaires sans infirmières ni médicaments. Bien entendu, la situation est encore plus dramatique au Sud, même si durant la guerre une offre alternative, humanitaire, a été mise en place. Surtout après 1991 quand la rébellion du Sud est devenue fréquentable, c'est-à-dire après la chute du régime prosoviétique de Mängestu qui a obligé le SPLM à abandonner ses bases arrière et ses soutiens logistiques en Éthiopie. Les rebelles ont dès lors pris des distances avec leurs perspectives socialistes et " oublié » leur formation à Cuba pour s'ouvrir aux lobbies humanitaires américains, notamment évangélistes. Dans le même temps, le Nord perdait de son intérêt stratégique avec la chute du Därg éthiopien et l'effondrement de l'Union soviétique d'autant que l'inqaz, le régime d'Omar al-Bashir, s'affichait de plus en plus comme une tête de pont de l'internationale islamiste émergente.

\section{Conclusions}

Fin 2003/début 2004, les premières missions humanitaires et d'évaluation des Nations unies découvrent au Darfour outre les destructions et les déplacements massifs de population - déjà plus de 1,5 million de personnes - une région totalement démunie, sans routes, sans eau, avec des hôpitaux délabrés. Une région oubliée du monde, surtout depuis l'application du boycott occidental au Soudan qui a radicalement réduit les moyens d'observation sur le terrain et interdit toute coopération en matière de développement. 
Sans le rappel au monde par des attaques éclairs et une farouche résistance de mouvements rebelles qui n'avaient pas imaginé l'extrême violence de la répression sans précédent qu'allait entraîner leur entrée en résistance, l'effondrement du Darfour aurait probablement perduré de longues années avec des actes d'humiliation et de spoliation toujours plus discriminants sur le plan ethnique. La perspective de paix Nord-Sud n'invitait pas la communauté internationale, pressée de reprendre langue avec le Soudan, à s'intéresser à un nouveau front intérieur ${ }^{20}$.

L'histoire contemporaine du Soudan nous enseigne que les demandes périphériques en faveur de plus de justice ne peuvent faire l'économie de la lutte armée ; il n'est pas pour autant certain que ces révoltes suffisent à ouvrir un plus grand éventail de perspectives de développement à long terme. Ainsi, malgré les appels à davantage d'autonomie et d'autodétermination, les régions périphériques semblent condamnées, au moins à court terme, à une double dépendance, tant vis-à-vis de la communauté humanitaire internationale qu'en ce qui concerne les capacités réelles de redistribution et de péréquation, mais aussi de gestion et d'encadrement, de l'État soudanais. Même le Sud, fort de la manne pétrolière, ne peut renoncer à une expertise exogène alors que lui font défaut plusieurs générations de cadres mais aussi de techniciens et d'employés compétents.

20. G. Prunier, Le Darfour. Un génocide ambigu, Paris, La Table Ronde, 2005. 